\title{
HAMALT : GENETICS BASED PEER-TO- PEER NETWORK ARCHITECTURE TO ENCOURAGE THE COOPERATION OF SELFISH NODES
}

\author{
T. C. Jermin Jeaunita ${ }^{1}$ T. C. Jermin Jersha ${ }^{2}$ \\ ${ }^{1}$ Department of Computer Science and Engineering, \\ PESIT Bangalore South Campus, Bangalore, India \\ jerminjeaunitatc@pes.edu \\ ${ }^{2}$ Department of Computer Science and Engineering, \\ P.S.V. Padmavathy Engineering College, Chennai, India \\ jerminjershatc@gmail.com
}

\begin{abstract}
Since its inception, Internet has grown tremendously not only in the size of its customers but also with the technology used behind to run it. For the well ex-istence and proper development of Peer-to-Peer Networks, all nodes in the overlay must be cooperative and donate their resources for any other peer. The paper dis-cusses the reason of peers being selfish, causes of selfish peers and the methods used so far to resolve selfish peers problem. A Genetic Algorithm based solution has been proposed in this paper that solves the selfish nodes problem in Peer-toPeer Networks and that also encourages the cooperation among all nodes in the overlay. An architecture HAMALT is proposed in this paper for disseminating altruism among the peers.
\end{abstract}

\section{KEYWORDS}

Altruism, Genetics, Kin Selection, Overlay, Peer-to-Peer Networks, Selfish Nodes.

\section{INTRODUCTION}

In peer to peer networks, a peer takes up the task of a master as well as slave; a client and a server; a requester and a provider. These peers are overlaid on the Internet for information sharing, hardware or software resource sharing, instant communication, on demand audio or video sharing and so on. For successful survival of a network that is made for such purpose, the well cooperation of all the peers in the network is required. But there will be some coward and selfish peers among the good peers that may stop the other peers in achieving the sole purpose of the network and threaten the proper subsistence of the network. Such peers are called as selfish nodes or free riders as they utilize the network for their benefit and sit inoperative otherwise and this practice of such nodes is termed as leeching.

Sundarapandian et al. (Eds) : ACITY, AIAA, CNSA, DPPR, NeCoM, WeST, DMS, P2PTM, VLSI - 2013 pp. 165-173, 2013. () CS \& IT-CSCP 2013

DOI : $10.5121 /$ csit.2013.3417 
Peers may be selfish for various purposes. In Peer-to-Peer networks which are used for various applications including social networking, a malicious peer may try to acquire the personal information of other peers to threaten them or for cost. When such misbehaving node is detected through the shared history mechanism[1] or trust mechanism[2], and when the node has learnt about this detection of its behavior, it can leave the network and join the same network with different identity. Such type of nodes is called as white-washers [3]. Some peer-to-peer networks punish such deviant nodes and when a new node joins the network and misinterpreted as a deviant node, the node might be discouraged [4]. Some Peer-to-Peer networks are built with titfor-tat strategy, where in a peer is allowed to access a resource based on its interaction with the corresponding node before the case. This leads to the discouragement of strange or new nodes after joining the network that obviously would not have had any interaction before with the peer it requested. This may inculcate selfishness in the new node which it can reflect later, when it receives a request. A node may also act selfish to increase its own utility and to reduce overheads [5]. They may go selfish about their limited battery power, bandwidth availability and transmission speed.

The free loaders can be treated in various ways. They can be deprived from accessing the network. Authors of reference [6] proposed an approach to punish the free riders by forbidding them to download files from the network if their utility value is lower than the size of the requested file. The requests submitted by the selfish nodes can be ignored or the TTL(Time to Live) of these request messages can be reduced[7]. A selfish peer if identified needs to pay a fine in the form of packet sending cost, which means the deviant node has to send some n number of penalty packets to continue in the network. A selfish node receives low QoS while a cooperative node receives high QoS.

The growth of selfish nodes worsens the continued existence of the network in various ways. It may also give wrong reputation to improve its incentive[8]. Some nodes are socially selfish such that they share resources to other peers which are only socially tied with them. Some peers may share with peers of stronger social tie than with weaker social tie. A selfish node may give wrong routing information or may devoid from forwarding routing packets, which may lead to longest path, more cost and wastage of resources. Peers may also deviate from forwarding data packets to any other peer proving individual selfishness. A selfish peer may utilize the services provided by other nodes in spite of concern of the resources of other peers. A selfish node may change its identity or neighbor to utilize the resources, so that it will not be identified as deviant node.

This paper proposes a new and interesting solution to resolve the free loaders problem by appropriately choosing the neighbor peers and keep the peers encouraging incessantly. Developing a framework to dole out altruism in the network will be a touchstone if the total evolutionary change of a network is also considered. The trait of a node to behave selfish is considered as evolved right from the self organization of the network. Hence for effective as well as efficient self organization of the network the problem is considered as a natural selection process of Genetics. Instead of considering socially tied peers as a drawback in the network, since they share information only with their socially tied peers, this paper considers the psychology of peers for forming a network of altruistic peers. Hence the architecture proposed is named as 'HAMALT' - a Hamilton Rule based Altruism Dissemination.

The remaining sections of the paper are organized as follows. Section 2 discusses the concepts of Genetics used for the proposed architecture. Section 3 describes the proposed HAMALT 
architecture. Mathematical proofs of the proposed lemmas that make the architecture are elaborated in section 4. Section 5 concludes the usefulness of the proposed framework.

\section{GENETICS}

Genetics is a scientific discipline that deals with the genes that are responsible for heredity of a living organism, and more specifically the physical or character trait of an organism. A gene can take various forms and each form of a same gene is called an allele. So literally an allele becomes responsible for the different observable or non observable traits present in any organism. Genes emanates phenes, as if genes are the biochemical instructions in the form of alleles in organisms while phenes are the observable characteristic of the organism itself. Hence the phenotype directly relates to the process of natural selection in organisms. Natural selection can be explained as the statistically consistent difference in reproductive success or fitness among phenotypes. Even more specific is the kin selection strategy of genetics that favors the reproductive success among relatives in-spite of an organism's own cost or survival.

\subsection{Kin Selection}

Genetics define kin selection as a theory of organisms helping their relatives than to any others, in spite of its own well being or survival. This leads to the transfer of a part or whole of the altruistic allele to the later generation of the selected kin.

\subsection{Inclusive Fiteness}

Inclusive fitness theory plays a vital role in learning the evolution of social behavior[9-12]. It is also said that inclusive fitness improves the phenotypic success of organisms by their altruistic social trait. Hence the genetic endowment of altruistic trait is considered as a predictor in the proposed mechanism. The goal of the proposal is to disseminate the fecundity of altruism among the peers of the network, so as to improve the coordination of all peers in the network.

\section{SYSTEM MODEL}

\subsection{Problem Description}

The system is considered as an overlay network with the peers ready to share their resources and the peers waiting to collect resources of their interest from other peers. If none other than few peers are ready to share their resources, the network either fails or very soon become a master/slave overlay network, and the goal of the Peer-to-Peer network may not be able to attain. Hence this paper proposes a framework called 'HAMALT' - a Hamilton Rule based Altruism Dissemination. The paper discusses solutions to keep all the peers active and encouraged for altruism.

\subsection{Parameters Used}

The parameters used in the proposed framework are tabularized in Table 1. 
Table 1: Parameters Description

\begin{tabular}{|c|l|}
\hline Parameters & \\
\hline $\mathrm{A} i$ & Inclusive fitness responsible for altruism \\
\hline $\mathrm{a} i$ & Individual fitness of any peer $i$ \\
\hline $\mathrm{V} i$ & $\begin{array}{l}\text { Average contribution of any peer } i \text { 's cluster of all two } \\
\text { hop peers }\end{array}$ \\
\hline $\mathrm{C} i$ & Contribution of peer $i$ to any other peer in the network \\
\hline $\mathrm{Cv} i$ & Summation of contributions of all one hop peers of peer $i$ \\
\hline $\mathrm{rij}$ & Degree of connectedness between peer $i$ and peer $j$ \\
\hline $\mathrm{L}$ & Loss to the donor \\
\hline $\mathrm{G}$ & Gain to the recipient \\
\hline $\mathrm{k}$ & Total number of chunks uploaded \\
\hline$\delta$ & Quantity of altruistic allele donated to the recipients \\
\hline $\mathrm{P} i$ & Representation of a peer with its nature as $i$ \\
\hline &
\end{tabular}

\subsection{HAMALT Architecture}

The proposed architecture with only two peers- one as donor and the other as recipient is modeled in Fig. (1). The working consists of two phases. In phase 1, every peer node calculates the altruistic fitness of itself using the fitness of other peers. The fitness is calculated using the contribution factor of the peer and its neighbors. Since the altruistic fitness depends on other peer's altruism, it is claimed that altruism of any peer encourages the other related and neighbor peers. The goal of Phase 2, is to spread altruism to the next generation. To keep all the nodes encouraged throughout the network's lifetime, it is not enough only to encourage the nodes to be altruistic. But at the same time, altruism itself must be transmitted from one generation to the next. 
Donor Peer

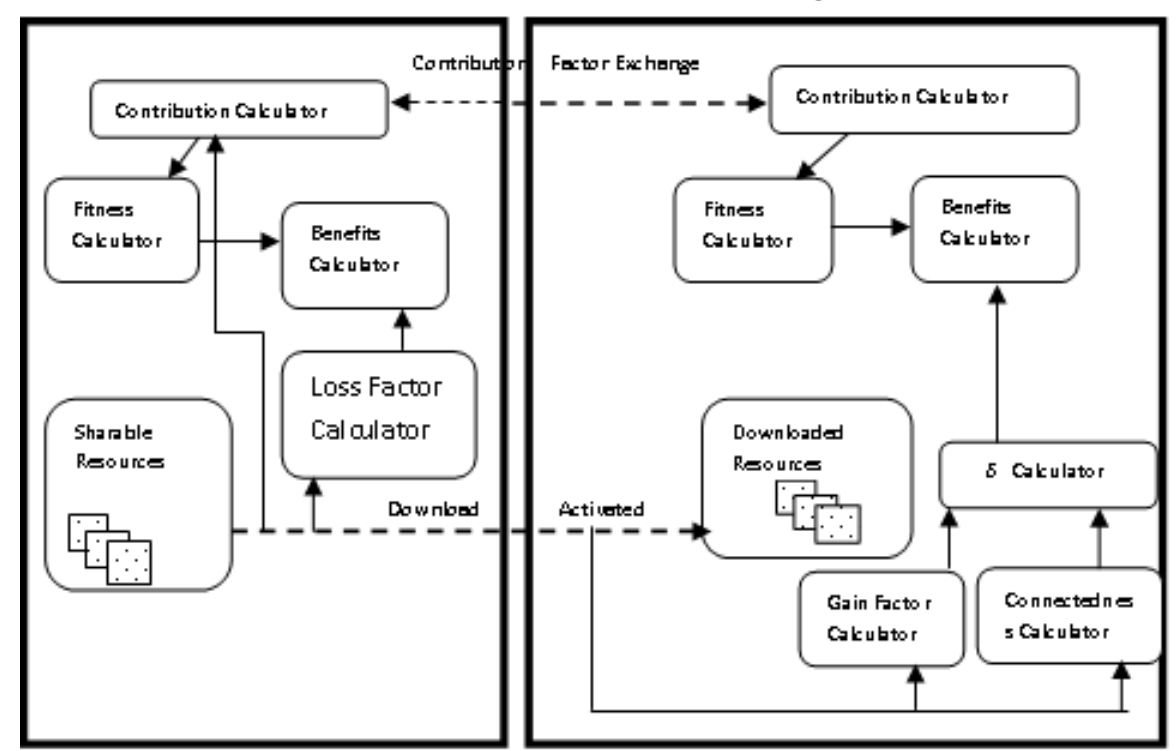

Fig.1. HAMALT Architecture with two peers- donor and recipient demonstrating the dependence of altruism and altruistic allele dissemination.

The contribution calculator calculates the contribution factor using the contribution factor exchange process. The allowances that can be provided for a donor node or if the node is selfish, necessary action to be taken is decided using the benefits calculator. The quantity of altruistic allele received by the recipients is also fed to the benefits calculator in case the node is a recipient so that the node is stimulated to be altruistic.

\section{SYSTEM DESIGN}

The two phases of the proposed framework are explained with mathematical proofs in the following subsections.

\section{1 Phase 1: Dependence of altruism}

Inclusive fitness depends on the peer's individual fitness which is a measure on its immediate neighbors or can be called as kin peers. Every peer tries to improve its own fitness for the survival in the network which will happen only if the peer possesses altruism. Inclusive fitness denoted as Ai of a peer $\mathrm{i}$ is found as

$$
\mathrm{A} i=\mathrm{a} i / \mathrm{V} i
$$

Where ai is the individual fitness of a peer $i$ and $V i$ is the average contribution of a peer i's cluster of all two hop nodes. The personal fitness or individual fitness of a peer is given by

$$
\mathrm{a} i=\mathrm{C} i+C \mathrm{v} i
$$

$\mathrm{Ci}$ is the contribution factor of peer $i$ to any other peer in the network and $\mathrm{Cv} i$ is the summation of contributions of all one hop peers of peer $i$, as shown in equ. (3). 


$$
C v i=\sum_{j} r i j . C j
$$

Where rij is the degree of connectedness between the peer $i$ and its one hop peers $j=1$ to $n, n$ is maximum number of one hop neighbors of peer $i$. The kin selection criteria also propose the selection of degree of relatedness. This degree of relatedness is shown in Fig. (2).

Lemma 1: Altruism of a peer depends on the altruism of the neighbor peers.

Proof: Equations (1) \& (2) show the dependence of altruism that discusses about the altruistic measure of every node which depends on the contribution of a neighbor peer to any other peer. When one peer's altruism depends on the altruism of another peer which is directly proportional to each other, obviously the peer also improves the altruistic measure of the neighbor peer. The degree of connectedness plays a major role to maintain the altruistic fitness and it is considered that $\mathrm{r} \leq 1$.

Dependence of altruism on other peer is demonstrated using Fig. (3). The figure shows an overlay of 7 peers. Fitness of peer $\mathrm{P} 1$ is calculated from the contribution factor of itself and contribution of its 1-hop peers (P2, P3, P4, P5). The contribution of peers up to two-hop (P2, P3, P4, P5, P6, $\mathrm{P7}$ ), shown in the figure helps to calculate the average contribution of the peer's population. The inclusive fitness $A i$ can be found from these two parameters.

Lemma 2: Personal fitness of a peer is reduced if it is not ready for contribution in the network.

Proof: If a request is neglected by a peer or if the individual altruistic factor $\mathrm{Ci}$ is constant for a specified duration, the individual contribution factor of a peer is reduced. A peer may utilize the altruistic nature of its neighbor and try to survive in the network without its own contribution as anyways the altruistic fitness of a neighbor increases its own too. But if allowed will invite more free riders. In order to avoid such scenario in the network, if a node is not ready to contribute or if the node goes to sleep mode, its contribution factor is reduced and it may lead to a chance of its ID added in the Neglected Peers List(NPL). Once if a node is added in the NPL, it is not allowed to access the benefits of the network.

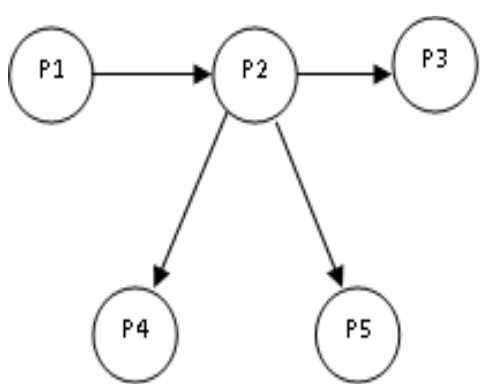

\begin{tabular}{|l|l|l|}
\hline Donor & $\begin{array}{l}\text { Recipient } \\
\text { Peer (i) }\end{array}$ & Connectedness \\
\hline P1 & P2 & 0.5 \\
\hline P2 & P3 & 0.5 \\
\hline P2 & P4 & 0.5 \\
\hline P2 & P5 & 0.5 \\
\hline P1 & P3 & 0.25 \\
\hline P1 & P5 & 0.25 \\
\hline P1 & P4 & 0.25 \\
\hline
\end{tabular}

Fig. 2. Coefficient of Connectedness: The directly connected peers are related by a factor of $r=0.5$ and 0.25 otherwise. 


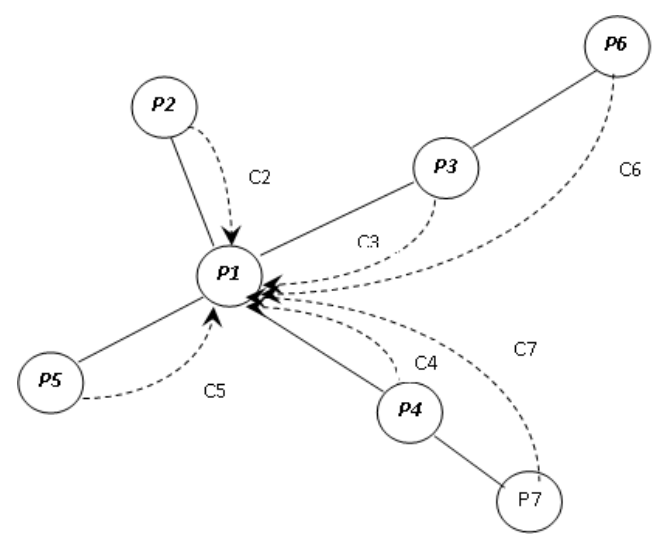

Fig. 3. Dependence of altruism on other peers

Lemma 3: A closely related peer encourages the altruism of its neighbor peers.

Proof: By Lemma 2, If the inclusive fitness of altruism is reduced, the framework notifies the intimates about the issue. The neighbor peers, find the relatedness with the selfish peer and sends an alert to its neighbor for its contribution to the network. The alert sent to the peer varies based on its relatedness with the other peer. If $r=0.5$, the alert carries the recommendation of the selfish peer to other peers, so that the so far selfish peer might get a request and improve its altruistic factor. This encourages the peer to come out of selfishness and act altruistic, as well as its chance of survival to benefit the network is improved.

\subsection{Phase 2: Spreading Altruism}

According to Hamilton's rule, altruism spreads to the recipients if the gain to the recipients is more than the loss to the donor, which is formulated as

$$
\mathrm{L}<\mathrm{rG}
$$

Where $\mathrm{L}$ is the loss factor to the donor, $\mathrm{r}$ is the connectedness of the recipient to the donor and $\mathrm{G}$ is the gain factor to the recipient. The formulation represented in equation (4), had also revealed that the donor's fitness is also improved by assisting their relative peers[13].

Lemma 4: The peer also donates its altruism to the recipient peer with the resources.

Proof: If the cost of each chunk $i$ of resource is $\mathrm{Li}$, the overall loss factor of a donor for one upload is given by

$$
L=\sum_{i=1}^{k} L i-\delta
$$

Where $k$ is the total number of chunks, $\delta$ is the altruistic allele donated to the recipients. If the gain of each chunk $i$ of resource is $\mathrm{Gi}$, the overall gain factor of a donor for one download is given by

$$
G=\sum_{i=1}^{k} G i
$$

and for simplicity, $\mathrm{Gi}$ is calculated from $\mathrm{Li}$ and it is given as

$$
G i=L i+\delta
$$

$\delta$ in equation (7) is the altruistic allele transferred from the donor to the recipient. 
Lemma 5: A peer with more gain is more altruistic in the next generation.

Proof: During the content discovery process a peer node searches for a resource for itself or for its relatives. When this peer identifies the resource and the same in $x$ peers where $\mathrm{x} \geq 2$, then there arises a tie for the proper peer as donor. Then a peer $\mathrm{P}$ is selected as a donor using the following criteria.

$$
A \max =\max (A p i) ; i=1 \text { to } n
$$

Where $\mathrm{n}$ is the maximum number of peers with the searched resource and Amax is the maximum fitness out of those $n$ peers.

The donor peer is $P d$,

such that $P d=P j$ if $A p j=A m a x$

Where Apj is the fitness of peer $P j$. Now $P d$ will act as the donor peer of next generation which is because of the altruistic allele $\delta$ in its gain factor $G$ that has improved its chance of being a donor.

Lemma 6: A peer with more fitness is recommended for benefits in the network.

Proof: Peers also maintain their interest on resources. When the loss factor $\mathrm{L}$ of a peer reaches or crosses a loss threshold, the peer is recommended for its resources of interest without any request by its relative peer to other peers in the network. This encourages every peer to be altruistic.

\section{CONCLUSION}

As the interest and demand for P2P networks accessing is growing day by day, the cooperation among nodes should attract more nodes to join the network. If the nodes are selfish to take up other peer's resources and not donate their resources, it may lead to the shutdown of the network or the network will be static. Even though many incentive based peer-to-peer resource sharing approaches exists, an approach based on kin selection is proposed in this paper. Since selfishness is a character of humanness or many other living organisms, and the solution to solve this trait in Genetics is proposed as a solution in selfishness problem in Peer-to-Peer networks. Hence this framework will avoid a peer to behave selfish, and encourage and disseminate altruism in all peers.

\section{REFERENCES}

[1] Stefano Arteconi, David Hales, and Ozalp Babaoglu: Greedy Cheating Liars and the ools Who Believe Them, ESOA 2006, LNAI 4335, pp. 161-175, Springer(2007).

[2] Qinghua Li, Wei Gao, Sencun Zhu, Guohong Cao: A routing protocol for socially selfish delay tolerant networks, Ad Hoc Networks, Elsevier, 2012, Volume 10, Issue 8, Pages 1619-1632 (2012).

[3] Murat Karakaya, Ibrahim Korpeoglu, Ozgur Ulusoy: Counteracting free riding in Peer-to-Peer networks, Computer Networks, Science Direct, 52: 675-694 (2008).

[4] Alex Friedman and Idit Keidar: Discouraging Selfishness in Lossy Peer-to-Peer Networks, Technion (2009)

[5] Idit Keidar Roie Melamed Ariel Orda: EquiCast: Scalable Multicast with Selfish Users, ACM, PODC (2006). 
[6] Landon P. Cox, Brian D. Noble: Samsara: Honor Among Thieves in Peer-to-Peer Storage, ACM, SOSP (2003).

[7] Alberto Blanc, Yi-Kai Liu, Amin Vahdat: Designing Incentives for Peer-to-Peer Routing, IEEE, INFOCOM (2005).

[8] Michael Sirivianos Xiaowei Yang Stanislaw Jarecski: Dandelion: Cooperative Content Dis-tribution with Robust Incentives, Usenix (2007).

[9] James A.R. Marshall: Group selection and kin selection: Formally equivalent approaches, Trends in Ecology and Evolution, Elsevier (2011).

[10] W. D. Hamilton: Innate Social Aptitude of Man: An approach from Evolutionary Genetics, Biosocial Anthropology. pp. 133-155. Wiley, New York. Hardin, G. (1968).

[11] Steven A Frank: George Price's Contributions to Evolutionary Genetics, Journal of Evolutio nary Biology, Elsevier (1995).

[12] B. Brembs: Hamilton's theory, Encyclopedia of Genetics, Academic Press (2001).

[13] (Online) www.wwnorton.com 